\title{
Investigation of the GaP/Si Interface by High-Resolution Scanning Transmission Electron Microscopy
}

\author{
A. Beyer, * J. Ohlmann, * M. Luysberg, ${ }^{* *} \mathrm{~K}$. Volz * \\ * Philipps-University Marburg, Department of Physics \& Materials Science Center, 35032 Marburg, \\ Germany \\ ** Ernst Ruska-Centre for Microscopy and Spectroscopy with Electrons, Forschungszentrum Jülich \\ GmbH, 52425 Jülich, Germany
}

Current developments in correcting not only the aberrations of the objective lens, but also of the condenser lens of transmission electron microscopes allow very high resolutions even in scanning transmission electron microscopy (STEM). Due to its z-contrast, high angle annular darkfield (HAADF) technique can provide information on a specimen's chemical composition, thus making it a perfect tool for the investigation of interfaces and crystal defects on atomic scale. We use HAADF to characterize gallium-phosphide $(\mathrm{GaP})$ grown on silicon $(\mathrm{Si})$. Because of its lattice constant similar to the one of $\mathrm{Si}, \mathrm{GaP}$ is promising as a buffer between the $\mathrm{Si}$ substrate and optical active layers like $\mathrm{Ga}(\mathrm{NAsP})$ for instance [1]. By combining the mature Si technology and the advantageous optical properties of III/V semiconductors a wide field of new applications becomes feasible, including high-efficient solar cells, high mobility n-channel layers and even a laser on Si substrate. During the heteroepitaxy of the polar III/V material on the nonpolar Si with a slight lattice mismatch of $0.3 \%$ (at room temperature) several defects may arise. The most common ones are antiphase domains, which develop at monoatomic steps on the silicon surface, and planar defects. By application of special growth conditions the defect density can be reduced to a minimum [2]. Nevertheless the quality and in particular charge neutrality of the interface is crucial for the performance of a device, therefore the investigation of the interface is of special interest.

GaP layers of thicknesses up to $120 \mathrm{~nm}$ were grown by metal organic vapour phase epitaxy (MOVPE) on (001) Si substrates with an intentional miscut of $0.1^{\circ}$ and $2^{\circ}$ into [110], respectively, to support the formation of doublesteps on the Si surface. For detection of any defects and interface investigations electron transparent specimens with viewing directions along the step edges and perpendicular to the step edges were prepared using mechanical polishing and argon ion milling. The samples were investigated in a double corrected JEOL JEM2200FS and a FEI Titan 80-300 in STEM mode utilizing HAADF technique resulting in z-contrast images.

In the acquired HAADF images the three present elements can be distinguished by their different intensities. This allows the investigation of the interface as well as antiphase boundaries atomically resolved, which can be seen in Fig. 1. To evaluate the chemical composition at the interface, intensity profiles were acquired across the interface. The intensity maxima were approximated by Gaussian peaks at the atomic positions (Fig. 2) and the fraction of silicon on the corresponding column was calculated and is depicted in the inset of Fig. 2. Five layers of intermixing of GaP with $\mathrm{Si}$ are observed which are more than expected for a charge neutral interface [3]. Moreover the $2^{\circ}$ off oriented samples show slightly different intermixing behavior compared to the samples with only $0.1^{\circ}$ miscut. 
The present contribution will show how HAADF imaging can be used to investigate defects as well as chemical composition and strain at interfaces on an atomic scale. With this knowledge structural quality of the layers and especially the interface can be improved even further.

\section{References}

[1] B. Kunert et al., Appl. Phys. Lett. 88 (2006) 182108.

[2] K. Volz et al., J. Crystal Growth 315 (2011) 37.

[3] W. A. Harrison et al., Phys. Rev. B 18 (1978) 4402.

[4] This research was supported by the German Science

Foundation in the framework of the European Graduate

College "elctron-electron-interactions in solids"

as well as by the Heisenberg program (KV).
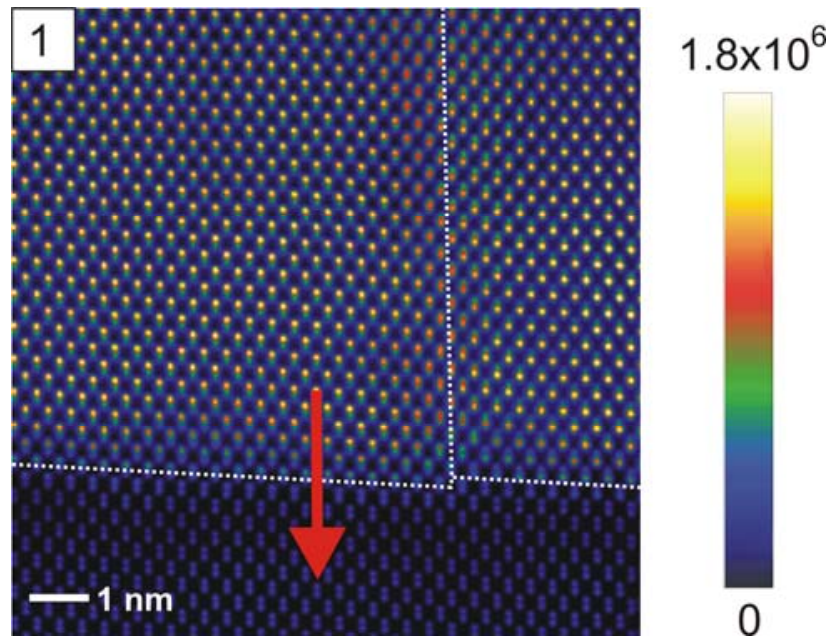

FIG. 1. HAADF image of GaP layer on silicon: The GaP/Si interface and a boundary between two domains of opposite polarity are visible and are marked by a dashed line. The orientation of the intensity profiles in Fig. 2 is indicated by the arrow.

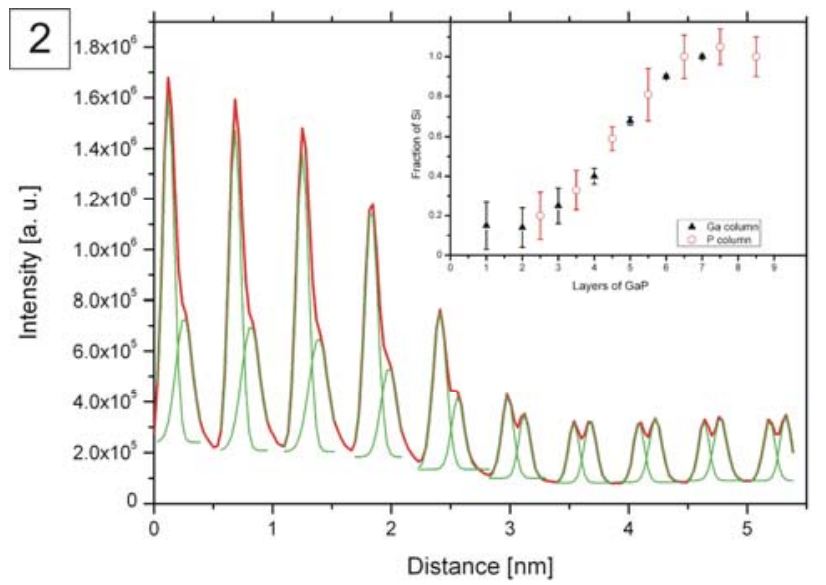

FIG. 2. Exemplary intensity profile across $\mathrm{GaP} / \mathrm{Si}$ interface (red line). The intensities were approximated by Gaussian peaks at the atom positions (green lines). The inset depicts the calculated fraction of silicon on the corresponding atomic column. 